\title{
DEVELOPMENT OF OPEN SOURCE HARDWARE IN ONLINE COMMUNITIES: INVESTIGATING REQUIREMENTS FOR GROUPWARE
}

\author{
R. Mies ${ }^{1, \bigotimes}$, J. Bonvoisin ${ }^{2}$ and R. Stark ${ }^{1}$ \\ ${ }^{1}$ Technische Universität Berlin, Germany, ${ }^{2}$ University of Bath, United Kingdom \\ $\triangle$ robert.mies@tu-berlin.de
}

\section{Abstract}

Open source hardware is hardware whose design is shared online so that anyone can study, modify, distribute, make, and sell it. In spite of the increasing popularity of this alternative IP management approach, the field of OSH remains fragmented of diverse practices seeking for settlement. This challenges providers of groupware solutions to capture the specific needs of open source product development practitioners. This contribution therefore delivers a list of basic requirements and verifies them by comparing offered functions of existing groupware solutions.

Keywords: open innovation, open source design, collaborative design, open source hardware, open source product development

\section{Introduction}

Open source hardware (OSH) is "hardware whose design is made publicly available so that anyone can study, modify, distribute, make, and sell the design or hardware based on that design." 1 It is an extension of the intellectual property management and product development approaches from open source software (OSS) into the world of physical objects. In this emergent field, like in the more settled field of OSS, transparency and free disclosure of product-related information intends to foster participative, democratic, community-based forms of product development where any interested person can get involved, regardless of his / her geographical or organizational background. We term these emerging practices of product development open source product development (OSPD $)^{2}$ in analogy with the term open source software development (used for example by Carillo and Okoli, 2008) built upon an ideal of geographically and hierarchically distributed work based on voluntary participation.

The concept of OSH has been "triggering many discussions and exploration ventures from academia to industry and to the government" ( $\mathrm{Li}$ and Seering, 2019), partly because it clearly branches out from standard practices of IP management in industry. So far, the production of OSH has been mostly limited to non-commercial sectors such as grassroots communities, hobbyist markets, NGOs and academia (Troxler, 2016). Nonetheless, small and medium enterprises have shown that it is possible to

\footnotetext{
${ }^{1}$ See "Open Source Hardware Statement of Principles 1.0" under URL: https://www.oshwa.org/definition/.

${ }^{2}$ Other researchers name this concept differently, e.g. open source design (Howard et al., 2012), open design (Aitamurto et al., 2015), open source innovation (Raasch et al., 2009). We however intentionally use OSPD because it unambiguously expresses the field we are interested in, whereas other terms have been used with very different meanings and would therefore be confusing in relation to this context.
} 
create monetary value around OSH, with potential advantages in terms of R\&D development costs and interesting service-related business routes ( $\mathrm{Li}$ et al., 2017). Early adopters of OSH practices are academics (Pearce, 2017) that OSH scientific equipment can be technically superior and less expensive than proprietary equipment.

Beyond business aspects, operating OSPD processes raises challenges regarding product data management (PDM) and collaborative product development environments (Bonvoisin and Boujut, 2015). Some initiatives have emerged over the last years to deliver groupware embedding such capabilities specifically tailored for $\mathrm{OSH}$, among which are Knowable.org (terminated), Wevolver.com, and Wikifactory.com. Groupware has been defined by Ellis et al. (1991) as "computer-based systems that support groups of people engaged in a common task (or goal) and that provide an interface to a shared environment." These groupware solutions aim to combine the successful PDM and management practices at play in OSS and those conventionally used in industrial closed source product development. So far, no dominant design has emerged and take up by OSPD practitioners is modest. Previous research from the authors showed that in the absence of generally acknowledged dedicated groupware recognized as meeting their needs, practitioners of OSH have learnt to re-purpose available groupware originally developed for open source software development (Bonvoisin et al., 2017b). This issue is salient since online platforms are a core element of any business based on open source, as Fjeldsted et al. (2012) observed.

The work presented here aims to contribute to the emergence of supporting groupware for OSPD by discovering requirements and providing a benchmark of groupware solutions currently used by OSPD practitioners. Partial insights in this direction have been delivered by Abhari et al. $(2016,2017)$ who developed a conceptual model of what they term "social product development" and developed a method to measure the affordances offered by corresponding supporting platforms. While these works mention some relevant technical features enabling co-development, they address the topic at a methodological meta-level and do not aim to deliver an exhaustive requirement set for such platforms. In contrast to this, the present paper elicits a set of requirements reflecting the needs of OSPD practitioners identified through a research method involving interviews and desktop research. Using inter-organisational collaborative development framework introduced by Lünnemann et al. (2016), we classify these requirements into four subsets covering four categories which are further elaborated in sub-section 2.1. Based on these requirements, the capacity of available groupware to support collaborative development of OSH products is evaluated through cumulative ratings. As a result, this study provides OSPD practitioners a comparison of different functions provided by relevant groupware solutions and delivers a set of verified requirements. The resulting profiles indicate that existing groupware solutions follow different approaches to facilitate OSPD. Looking forward, this comparison is first of all hoped to ease the selection of suitable groupware solutions for practitioners. In the longer term, the collected set of requirements provides a baseline for further future development of groupware solutions.

\section{Groupware for open source product development}

\subsection{Definitions}

The transformation of the open source logic from bits to atoms, or alternatively, the process of OSPD refers to the development of OSH products performed in a collaborative development process allowing the participation of any interested person (Bonvoisin et al., 2018). OSPD is characterized by the simultaneity of two forms of openness (Huizingh, 2011): product openness (the "source" of the hardware is made publicly available) and process openness (any interested person can take part in the development process). Generally, OSPD processes are relevant for complex products, whose definition requires the collaborative integration of inputs from multiple contributions. This, however, does not exclude the existence of OSPD processes for simple products. Consequently, for groupware solutions, supporting OSPD means supporting both product openness (the capacity to document products and share product documentation) and process openness (the capacity to participate in a product development process). 
Product development is defined as the set of intellectual activities processed in order to move from a product idea to the release of a fully defined and detailed product documentation that can be used for production. Product development is therefore to be delineated from production, the latter being the physical realisation of the plans defined in the former. This contribution studies groupware for OSH communities in order to support product development only, and not production. It also excludes aspects like networking of local workshops or material marketplaces.

Furthermore, the term "hardware" in Open Source Hardware refers to any form of discrete manufactured tangible products, be it electronic hardware, mechanical hardware or even textile. This contribution focuses on groupware that supports the collaborative development of all kinds of hardware. It is however restricted to discrete manufacturing and therefore excludes products of food and process industries such as yoghurt, cement, chemicals or plastic compounds.

Lastly, it should be noted that OSH is not DIY, although both OSH and DIY designs are two interwoven phenomena. Not all OSH is meant for DIY production (as per Boisseau et al. (2017) it may also be for the industrial context). Not all DIY products are open source as well. DIY instructions published in magazines may not comply with the requirements of the above-mentioned OSH definition by the OSHWA regarding licensing terms. The type of groupware studied here therefore does not cover exchange platforms for DIY instructions such as Instructables ${ }^{3}$.

Groupware for the development of complex OSH products supports interaction of geographically dispersed individuals. In the context of computer-supported collaborative work (CSCW), design is mainly characterised by the interaction between individuals and artefacts or the interaction between different individuals (Saad and Maher, 1996). This contribution looks at the latter with a focus on activities of collaboration. Collaboration can be further distinguished as intra- or interorganisational. Lünnemann et al. (2016) propose a definition of collaboration which considers both perspectives as per the following four categories (Lünnemann et al., 2016 refer to Steinheider, 2001):

- Communication - exchange of information between individuals;

- Coordination - definition and assignment of tasks and activities towards joint outcomes;

- Knowledge integration - provision and securing of recordable information for further use;

- Information logistics - targeted and regulated provision and recording of data and information in networks.

\subsection{Method for requirements discovery}

In order to gather and analyse practitioners' needs regarding groupware solutions, an initial list of requirements was extracted from interviews with founders and contributors of 23 selected OSPD projects as a first step. The interviewees were guided through a pre-defined list of questions on the general use of software tools and functions (such as "Do you use an online design platform?" or "Which tools do you use to communicate with other community members?"), as well as functions of an ideal online design platform ("What does the perfect platform look like to you?"). These lasted between 10 and 20 minutes (see Bonvoisin et al., 2017b for the full interview protocol). Notes that were taken during the interviews along with transcripts created were then used to write up minutes and aggregated to overviews of findings. This allowed the authors to derive an initial set of requirements that were formulated to allow for a binary scale of "yes" or "no". Following on, desktop research was used for the authors to get better accustomed with the requirements that were extracted from the interviews. Thereby the practical usage of groupware solutions by OSPD projects that was publicly visible was examined closely. This allowed the refinement and extension of the initial list of requirements according to real conditions which had to be separated from idealised depictions of the interviewees. Last, the requirements were verified by assessing groupware solutions for OSPD (which will be selected in the next sub-section) as to whether they fulfil them. The following Figure 1 illustrates the three steps of the methods for requirements discovery:

\footnotetext{
${ }^{3}$ See URL: https://www.instructables.com.
} 


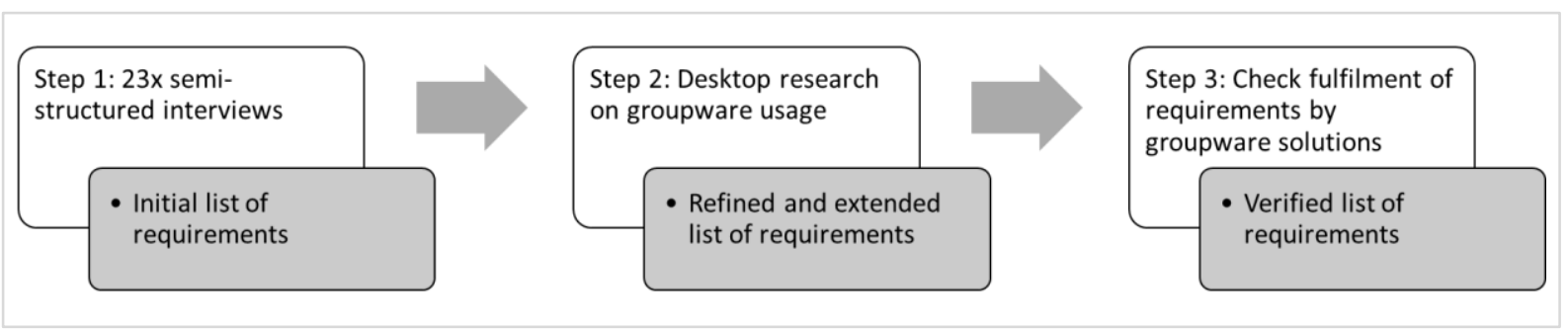

Figure 1. Three steps of the methods for requirements discovery

\subsection{Selection of groupware solutions}

Groupware solutions assessed in this contribution were selected based on their adoption in the field of OSPD, approached by the number of OSPD projects that utilise them. Groupware solutions candidates were identified through the OSPD projects referenced in the Open Hardware Observatory's (OHO) Project Directory ${ }^{4}$ created in the context of earlier research from the authors in collaboration with relevant actors in the field of OSH (e.g. Bonvoisin et al., 2017a). To the knowledge of the authors, this database provides the most extensive publicly available list of 223 projects for complex, mechanical OSH. The list was compiled and extended over time through various searches, interviews and exchanges with practitioners and experts and case studies from literature. As part of this data collection, the authors on the other hand compiled a list of 65 websites that were hosting OSPD projects or OSH-related documentation (e.g. CAD files, instructions, bill of materials). The list of 65 identified websites was screened for fulfilment of the following criteria:

1. Availability of the website (the website is not down, temporarily unavailable, or terminated)

2. Accessibility of projects on the website or to the documentation of products in general

3. Hosting of relevant projects (i.e. comprehensive focus on product development and not mere DIY production, very simple products / gimmicks, or single-3D parts for printing; availability of working prototypes; availability of technical drawings)

4. Hosting of a significant number of projects that are referenced in the OHO Project Directory $(>10)$

Out of the 65 screened websites, $9 \%$ were excluded because they did not comply with criterion 1 ; $15 \%$ were excluded because they did not comply with criterion 2; 66\% of the websites were excluded as a result of failing to comply with criteria 3 and 4 which were checked simultaneously. As a result, from the 65 websites, five groupware solutions have been considered as relevant for this study: GitHub (a hosting provider for distributed versioning control system Git), GrabCad (a platform for managing and sharing CAD files), Wevolver (a platform for sharing OSH), Wikifactory (a platform for social product development), and MediaWiki (the free and open source wiki engine for Wikipedia).

The distribution of projects along the identified groupware solutions is depicted in Figure 2 against a measure of the openness of these projects. This measure is a 6-point scale based on the Open-o-Meter $(\mathrm{OoM})^{5}$ previously developed by the authors (see Bonvoisin and Mies, 2018). A score of 6 indicates maximal openness and a score of 0 indicates zero openness. The resulting profiles of the first three groupware solutions (from the left side) resemble a normal distribution around the median OoM rating of four. The figure does not indicate any relationship between the choice of the groupware solutions and the level of openness. The selected groupware solutions seem to facilitate a great diversity of projects from low to high openness ratings.

\footnotetext{
${ }^{4}$ See URL: https://en.oho.wiki/wiki/The_OHO_Product_Directory.

5 The Open-o-Meter is a simple scale where a product gets one point for each of the following aspects: design files are published; assembly instructions are published; a bill of materials is published; a contribution guide is published; published files are shared in original format; all this information is published under a license allowing commercial reuse.
} 


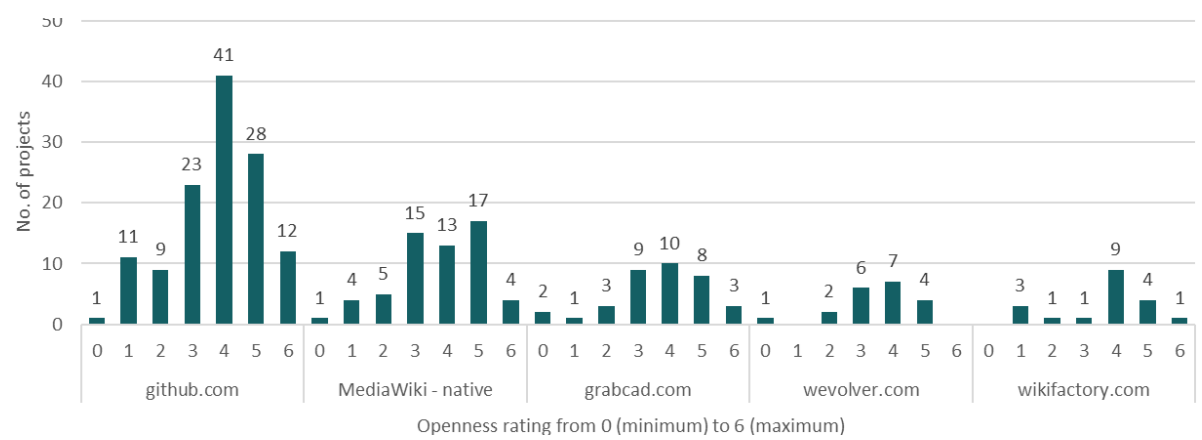

Figure 2. Distribution of projects for five groupware solutions according to openness ratings

\section{Results}

This section discusses the identified requirements along the four categories of collaboration used by Lünnemann et al. (2016). The formulated requirements were numbered and are listed in Appendix A.

\subsection{Communication}

Considering the common decentralized structures of OSH communities (see Ye and Kishida, 2003), communication in the context of OSH should support community management and user interactions. This comprises of the following basic requirements:

- User showcasing. Contributors have a user profile where they can showcase their contributions to public online repositories (storage locations which contain and organise files) on the groupware (given the context, public repositories will be referred to as "repositories" only). Their profile can give an idea of the volume and when they made contributions to repositories. Other users can then view their profile and eventually follow it. $R 1$

- Social networking. The groupware embeds the standard social networking features such as user following, repository following, project or contribution liking, and interconnection with other social networks. $R 2$

- Annotation and commenting. There is the possibility to have content related discussions, that is, to comment on selected content in a repository. At best, not only can files be attached with a discussion thread, but also contents of the files such as text section (as in MS Word for example) or a CAD feature (CAD annotation) can also be attached. $R 3$

- Communication channels. The groupware offers multiple (not content related) communication channels for public and private, as well as synchronous and asynchronous communication (such as messenger chats, forums, mailboxes). $R 4$

\subsection{Coordination}

Coordination in OSPD is considered by Huizingh (2011) as process openness and can be translated into the following basic requirements:

- Getting started. Essential project resources and contacts need to be easily accessible to new developers. The groupware can push-start process openness by facilitating projects to upload a contributing guide i.e. in the root directory of a project that encourages outside participation and defines how contributions are submitted and handled. $R 5$

- Task management. This can be delivered in the form of an issue tracking system to organise and prioritise tasks, or a Kanban board to visualize task lists and workflows, among other possibilities. At best, the declaration and the discussion of issues is opened for any user and their management is restricted to the development team. $R 6$

- Requirements management. There is the possibility to express, record, structure and manage requirements of the product to be developed. Track can be kept on the extent to which the current state of the product conforms to the recorded requirements. $R 8$ 
- Write-access. Only the owners of a repository have write-access to it.

An owner is considered either the person who created the repository or other persons that have been granted as owners by the initial owner. Integrating a modular brick from one repository to another does not grant the owner of the latter access on the contents of the former. In order to get access, the owner of the latter must fork the former. $R 9$

- Unlimited fork right. Any registered user has the unrestricted right to fork a repository. Forking a repository results in the creation of a new repository filled out with the contents of the forked repository. The forking person is the owner of the newly created repository and has therefore write-access. Forking a repository does not grant the forking person with write access on the original repository. $R 10$

- Submission mechanism. The owner of a fork can suggest to the owner of the repository the fork is issued from to adopt the changes made in the fork. The owner of the original repository has the right to either adopt or decline the suggested changes. In the case where he or she adopts the suggested changes, the modified contents in the fork are ported in the original repository. In case where he or she declines the changes, he or she has the possibility to request the owner of the suggested changes to rework them. R11

- Full history versioning. Each modification made to the content of a repository is recorded so it is always possible to know who did what, and when he or she did it. Versioning data may include: the content of the changes (before / after), the date of the changes, a reference of the person who made the changes, a justification for the changes (comment). This allows the status of a repository at any time to be retrieved and the statuses of a repository at two different times to be compared. $R 12$

\subsection{Knowledge integration}

Knowledge in OSH is continuously explicated through OSH documentation. This process integrates knowledge as per the follows requirements:

- "Source" of hardware products. The openness of OSH depends on four freedoms, each of them setting different requirements on the content and the format of the data to be shared publicly:

- Freedom to study (i.e. the right to access sufficient information to understand how the piece of hardware works and to retrace the underlying design rationale) can be supported by the publication of schematics, 2D or 3D CAD files as well as textual information.

- Freedom to modify (i.e. the right to edit the product definition documents and to modify or develop the product further for any purpose) can be supported by the publication of all documents in their original editable format.

- Freedom to make (i.e. the right to use the product definition documents in order to make - in other words to produce, to manufacture - the piece of hardware) can be supported by the publication of bill of materials and assembly instructions.

- Freedom to distribute (i.e. the right to give or sell the documents and the physical products fabricated with the help of these documents) is allowed by the publication of all documents under a licence which grants free redistribution including for commercial purposes. $R 13$

This means that textual contents such as general project information including the embedding of image files in the text is supported. R14

In order to fulfil this requirement, the groupware needs to support the sharing of export as well as original file formats. $R 15$

There are different ways to implement this. The groupware may either allow the user to upload documents or spreadsheets or provide an online form that complies with standards such as VDI 4500, part 4. $R 16 / R 17$

Knowledge integration in OSH also occurs along the product lifecycle which is reflected by the supported project scope:

- Lifecycle phases. Product development encompasses different types of activities which can be separated in "product ideation" and "narrowly" defined "product development". In product 
ideation, different product concepts are proposed to fit with a defined set of product requirements, these concepts are discussed and the more promising ones are chosen. In product development, the focus lies in the generation of fully defined product documentation enabling the production of the chosen product concept. Some groupware solutions also integrate "manufacturing and process planning". $R 18 / R 19$

\subsection{Information logistics}

Information logistics in OSH first of all, requires as a general pre-condition, ease of access, which is supported through the following requirements:

- Unrestricted view access. Any unregistered user can list and open all files of a repository without any restriction. The groupware offers in-browser display of any type of content listed above which is stored in a non-proprietary or free of charge format. $R 21$

- Product architecture. The architecture of a physical product can be represented by a tree. The product documentation should reflect this structure in order to ease information storage and recovery. At best, the groupware allows managing structures like CAD assemblies and skeletons. $R 22$

- Single entry point. Depending on which product-related documentation is stored, the structure should allow information reuse and avoid redundancies. The groupware should allow to reflect a whole system, a full assembly or a simple component. $R 23$

Another central aspect of information logistics in OSH pertains to software architecture as per the following requirements:

- Open source. In the effort of approaching the ideal of a complete, open source tool chain, the groupware should be itself open source software. $R 24$

- Independence. In order to avoid dependence to any service provider, the groupware should be a software solution anyone can deploy on his/her own personal computer or server. This avoids data to be controlled by a central actor as the sole owner of the current version of all data. $R 25$

- Decentricity. In order to promote data resilience and decentral organisation of work, the groupware should be based on a $\mathrm{P} 2 \mathrm{P}$ or server-client architecture allowing any contributor to get a copy of the project data. This ensures that the data remains available if a groupware instance breaks down. $R 26$

- Interconnectedness. If wished by its provider, each instance of the groupware can be embedded in a network making certain searchability of repository content. That is, the decentral network of groupware instance can be browsed and searched as if it were a central platform. $R 27$

This means that data sets are automatically federated.

\section{Comparison of groupware solutions}

The five selected groupware solutions for OSPD (see section 2) have been assessed in terms of whether they fulfil the 27 requirements from the previous section. Each time a requirement was met by the groupware solution, one point was awarded. The detailed assessment can be found in Appendix A. The amount of points for each category are listed in the following Table 1:

Table 1. Comparison between groupware for open source product development as per amount of provided functions

\begin{tabular}{|l|c|c|c|c|c|c|}
\hline Category & $\begin{array}{c}\text { Max. } \\
\text { score }\end{array}$ & GitHub & $\begin{array}{c}\text { MediaWiki } \\
\text { (native) }\end{array}$ & GrabCad & Wevolver & Wikifactory \\
\hline 1. Communication & $(4)$ & 2 & 2 & 3 & 3 & 3 \\
\hline 2. Coordination & $(8)$ & 7 & 2 & 2 & 2 & 3 \\
\hline 3. Knowledge integration & $(8)$ & 4 & 3 & 4 & 6 & 7 \\
\hline 4. Information logistics & $(7)$ & 4 & 5 & 3 & 3 & 3 \\
\hline Total & $\mathbf{( 2 7}$ & $\mathbf{1 7}$ & $\mathbf{1 2}$ & $\mathbf{1 2}$ & $\mathbf{1 4}$ & $\mathbf{1 6}$ \\
\hline
\end{tabular}


Comparing the varying profiles of each groupware solution illustrates that they follow very different approaches to facilitate OSPD. In other words, there is no dominant design. This is also illustrated by the fact that none of the groupware solutions reach the maximum possible score of 27.

The winner of this comparison is the groupware solution GitHub with 17 out of 27 possible points closely followed by Wikifactory with one point less. GitHub has the best rating on coordination, whereas Wikifactory scores best on facilitating communication and knowledge integration. The nextbest score with 14 out of 27 possible points is achieved by Wevolver with a very similar profile to Wikifactory in terms of strengths and weaknesses. They both tend to focus more on broadcasting of product designs than on coordination and information logistics. MediaWiki and GrabCad come last in this comparison with 12 out of 27 possible points. However, MediaWiki scores highest on information logistics which is viewed as a very important aspect by many practitioners (with regard to the aspects of open source and independence). This partly explains why MediaWiki is widely utilized in OSH.

Out of the compared solutions, no groupware follows a strong focus on product modelling. An integration of modelling and simulation functions by future groupware solutions would certainly be of great value. As well, a Kanban board for issues is only provided by GitHub, even though this is an essential feature to manage projects. Also, it was found that none of the groupware solutions provide comprehensive public/private synchronous/asynchronous communication channels, functions for requirements management, or data federation. These would be important aspects to be implemented in the future.

\section{Conclusion}

In this contribution, a list of 27 binary requirements representing the needs of OSPD practitioners was elicited. These requirements were verified by assessing them against five widely used groupware solutions for OSPD. The formulation of requirements for binary scales allows higher replicability of the findings but also limits the granularity of the assessment. It should also be noted that more than two thirds of the interviewees were male and of European citizenship. Only about one fifth of the interviewees were women and about one fifth of the interviewees were North-American citizens. This limits the transferability of the results in terms of gender diversity and beyond high-income economies, factors that should be considered in future research.

The comparison of the solutions shows no clear champion, but different strengths and weaknesses which reflect different approaches that have found their place in the field of OSH to facilitate its progress. Notwithstanding, there is plenty of room in the coming years to extent existing groupware solutions, especially in terms of open source-based, independent solutions. Interestingly, big players like the MediaWiki project just as start-ups like Wikifactory and Wevolver have found their niches to have an impact on OSPD. Yet, as this comparison has shown, the most relevant aspect of open source development is to support coordination which makes GitHub just as relevant a groupware for the development of hardware, as for free and open source software. We expect that future expansion of the field of OSH will create a growing market for new groupware solutions for which the requirements discovered within this contribution provide a baseline.

\section{Acknowledgement}

The research was conducted in the frame of the research project "Open! - Methods and tools for community-based product development" and was funded by the DFG (Deutsche Forschungsgemeinschaft, grant STA 1112/13-1).

\section{References}

Abhari, K., Davidson, E. and Xiao, B.S. (2016), "Taking open innovation to the next level: a conceptual model of social product development (SPD)", Twenty-second Americas Conference on Information Systems, San Diego, 2016.

Abhari, K., Davidson, E. and Xiao, B.S. (2017), "Co-innovation platform affordances: Developing a conceptual model and measurement instrument”, Industrial Management \& Data Systems, Vol. 117 No. 5, pp. 873-895. https://doi.org/10.1108/IMDS-05-2016-0156

Aitamurto, T., Dónal, H. and Sofia, H. (2015), “The Open Paradigm in Design Research”, Design Issues, Vol. 31 No. 4, pp. 17-29. http://dx.doi.org/10.1162/DESI_a_00348 
Boisseau, É, Omhover, J., and Bouchard, C. (2017), "Open-design: A state of the art review", Design Science, 4, E3. https://doi.org/10.1017/dsj.2017.25

Bonvoisin, J. and Boujut, J.F. (2015), "Open design platforms for open source product development: current state and requirements", Proceedings of the 20th International Conference on Engineering Design (ICED15), Vol 8: Innovation and Creativity, Milan, Italy, 27-30.07.15, pp. 11-22.

Bonvoisin, J. et al. (2017a), "What is the 'Source' of Open Source Hardware?", Journal of Open Hardware, Vol. 1 No. 1, p. 5. http://doi.org/10.5334/joh.7

Bonvoisin, J. et al. (2017b), "Current state of practices in open source product development", Proceedings of the 21st International Conference on Engineering Design (ICED17), Vol. 2: Design Processes, Design Organisation and Management, Vancouver, Canada, 21-25.08.17, pp. 111-120.

Bonvoisin, J. and Mies, R. (2018), "Measuring Openness in Open Source Hardware with the Open-o-Meter", Procedia CIRP, Vol. 78, pp. 388-393. https://doi.org/10.1016/j.procir.2018.08.306

Bonvoisin J. et al. (2018), "How participative is open source hardware? Insights from online repository mining", Design Studies, Vol. 4 No. 19, pp. 1-31. https://doi.org/10.1017/dsj.2018.15

Carillo, K. and Okoli, C. (2008), "The Open Source Movement: A Revolution in Software Development", Journal of Computer Information Systems, Vol. 49 No. 2, pp. 1-9. https://doi.org/10.1080/08874417. 2009.11646043

Ellis, C.A., Gibbs, S.J. and Rein, G. (1991), "Groupware: some issues and experiences”, Communications of the ACM, Vol. 34 No. 1, pp. 39-58. http://doi.org/10.1145/99977.99987

Fjeldsted, A. et al. (2012), “Open source development of tangible products", In: DS 71: Proceedings of NordDesign 2012, the 9th NordDesign conference, Aarlborg University, Denmark. 22-24.08.2012.

Howard, T.J. et al. (2012), "Open Design and Crowdsourcing: maturity, methodology and business models", Proceedings of the 12th International Design Conference DESIGN 2012, pp. 181-190.

Huizingh, E.K. (2011), "Open innovation: State of the art and future perspectives”, Technovation, Vol. 31 No. 1, pp. 2-9. https://doi.org/10.1016/j.technovation.2010.10.002

Li, Z. et al. (2017), "Why Open Source? Exploring the Motivations of Using an Open Model for Hardware Development", In: ASME 2017 International Design Engineering Technical Conferences and Computers and Information in Engineering Conference, 6-9 August, 2017, Cleveland, Ohio, USA.

Li, Z. and Seering, W. (2019), "Does Open Source Hardware Have A Sustainable Business Model? An Analysis of Value Creation and Capture Mechanisms in Open Source Hardware Companies", In: Proceedings of the Design Society: International Conference on Engineering Design Vol. 1 No. 1, pp. 2239-2248. Cambridge University Press.

Lünnemann, P. et al. (2016), Zukunft der unternehmensübergreifenden Kollaboration, [online] Fraunhofer IPK, CONTACT Software, VDI, Berlin Available at: https://www.ipk.fraunhofer.de/fileadmin/user_upload/ IPK/geschaeftsfelder/vpe/Zukunft_der_unternehmensuebergreifenden_Kollaboration.pdf

Pearce, J.M. (2017), "Emerging Business Models for Open Source Hardware”, Journal of Open Hardware, Vol. 1 No. 1:2, pp. 1-14. https://doi.org/10.5334/joh.4

Raasch, C., Herstatt, C. and Balka, K. (2009), "On the open design of tangible goods", $R$ and D Management, Vol. 39, pp. 382-393.

Saad, M. and Maher, M.L. (1996), "Shared understanding in computer-supported collaborative design", Computer-Aided Design, Vol. 28 No. 3, pp. 183-192. https://doi.org/10.1016/0010-4485(95)00025-9

Steinheider, B. (2001), "Supporting the co-operation of R\&D-teams in the product development process", 5th Conference on Engineering Design and Automation. Las Vegas, Nevada. August, 2001.

Troxler, P. (2016), “Offene Freie Technik: Was Ist Offene Freie Technik (Open Source Hardware)?”, Die Welt Reparieren Open Source Und Selbermachen Als Postkapitalistische Praxis, pp. 85-92.

Ye, Y. and Kishida, K. (2003), "Toward an understanding of the motivation Open Source Software developers", Proceedings of the 25th international conference on software engineering, IEEE Computer Society, Portland, Oregon, May 3-10, 2003, pp. 419-429. http://doi.org/10.1109/ICSE.2003.1201220 


\begin{tabular}{|c|c|c|c|c|c|c|}
\hline \multicolumn{7}{|c|}{ 1. Communication } \\
\hline \multirow{4}{*}{$\begin{array}{l}\text { Community } \\
\text { management } \\
\text { and user } \\
\text { interactions }\end{array}$} & R1 - The groupware allows contributor showcasing & 1 & 0 & 1 & 1 & 1 \\
\hline & R2 - The groupware supports social networking & 1 & 0 & 1 & 1 & 1 \\
\hline & $\begin{array}{l}\text { R3 - Repository contents can be attached with a } \\
\text { discussion/comment thread }\end{array}$ & 0 & 1 & 1 & 1 & 1 \\
\hline & $\begin{array}{l}\mathrm{R} 4-\text { The groupware provides public/private } \\
\text { synchronous/asynchronous communication channels }\end{array}$ & 0 & 0 & 0 & 0 & 0 \\
\hline
\end{tabular}

\section{Coordination}

openness

(accessibility)

\section{Knowledge Integration}

R5 - The groupware supports the uploading of a contributing guide

R6 - The groupware offers public issue lists

R7 - The groupware offers a Kanban board for issues

$\mathrm{R} 8$ - The groupware allows managing requirements

R9 - Only the owners of a repository have write-access

R10 - Any registered user can fork any repository

R11 - The groupware embeds a mechanism allowing

suggesting changes to a repository owner

R12 - The groupware allows retrieving full versioning information (who did what and when)

\begin{tabular}{|c|c|c|c|c|c|c|}
\hline \multicolumn{7}{|c|}{ 3. Knowledge Integration } \\
\hline \multirow{5}{*}{$\begin{array}{l}\text { Product } \\
\text { openness } \\
\text { (transparency) }\end{array}$} & $\begin{array}{l}\text { R13 - The groupware supports the storage of textual } \\
\text { information (with embedded media) }\end{array}$ & 1 & 1 & 0 & 1 & 1 \\
\hline & $\begin{array}{l}\text { R14 - The groupware supports the storage of } 2 \mathrm{D} \text { and 3D } \\
\text { schematics }\end{array}$ & 1 & 0 & 1 & 1 & 1 \\
\hline & $\begin{array}{l}\text { R15 - The groupware supports the storage of bills of } \\
\text { materials }\end{array}$ & 0 & 0 & 0 & 1 & 1 \\
\hline & $\begin{array}{l}\text { R16 - The groupware supports the storage of assembly } \\
\text { instructions }\end{array}$ & 0 & 0 & 0 & 1 & 1 \\
\hline & $\begin{array}{l}\text { R17 - The groupware allows identifying the terms (li- } \\
\text { cense) under which each piece of information is shared }\end{array}$ & 1 & 1 & 0 & 1 & 1 \\
\hline \multirow{3}{*}{ Project scope } & R18 - The groupware supports ideation mechanisms & 0 & 0 & 1 & 0 & 0 \\
\hline & R19 - The groupware supports product development & 1 & 1 & 1 & 1 & 1 \\
\hline & $\begin{array}{l}\text { R20 - The groupware supports manufacturing and } \\
\text { process planning }\end{array}$ & 0 & 0 & 1 & 0 & 1 \\
\hline \multicolumn{7}{|c|}{ 4. Information logistics } \\
\hline \multirow{3}{*}{$\begin{array}{l}\text { Ease of access } \\
\text { (general } \\
\text { conditions) }\end{array}$} & $\begin{array}{l}\text { R21 - The groupware does not require any condition for } \\
\text { viewing the contents stored in its repositories }\end{array}$ & 1 & 1 & 0 & 1 & 1 \\
\hline & $\begin{array}{l}\text { R22 - The groupware supports the structuration of } \\
\text { documentation according to the product architecture }\end{array}$ & 1 & 1 & 1 & 1 & 1 \\
\hline & $\begin{array}{l}\text { R23 - The groupware allows navigating into all the } \\
\text { contents of a repository from a single entry point }\end{array}$ & 1 & 1 & 1 & 1 & 1 \\
\hline \multirow{4}{*}{$\begin{array}{l}\text { Software } \\
\text { architecture }\end{array}$} & R24 - The groupware is open source software & 0 & 1 & 0 & 0 & 0 \\
\hline & $\begin{array}{l}\text { R25 - Anyone can create their local instance of the } \\
\text { groupware and host project repositories }\end{array}$ & 0 & 1 & 0 & 0 & 0 \\
\hline & $\begin{array}{l}\text { R26 - Every contributor of a repository can host a copy } \\
\text { of a repository they are contributing to }\end{array}$ & 1 & 0 & 1 & 0 & 0 \\
\hline & $\begin{array}{l}\text { R27 - Each instance of the groupware and its content } \\
\text { can be known by the other instances }\end{array}$ & 0 & 0 & 0 & 0 & 0 \\
\hline \multicolumn{2}{|l|}{ Total } & 17 & 11 & 12 & 14 & 16 \\
\hline
\end{tabular}

